\title{
We need greener conferences
}

\author{
Attending conferences is an essential part of scientific careers; yet, travelling - particularly by air - can often \\ be an individual's single largest contribution to their carbon footprint. With increasing calls to substantially cut \\ emissions by as early as 2020, compromises must be made to safeguard the health of our planet.
}

S ummer is the season of travelling, and for scientists and editors, much of the travelling during these months is work-related. For example, this editorial team will have attended 12 conferences during the summer, mostly international meetings involving air travel. Conferences are an important part of our jobs and of the life of academics, providing opportunities to interact with colleagues and to stay up-todate with what's at the forefront of each field, yet such travelling has a significant environmental cost. For example, a 2014 report from UC Santa Barbara estimated that around one-third of the university's carbon emissions resulted from workrelated air travel by faculty and staff. An earlier report from the University of Oxford estimated that $7 \%$ of its $\mathrm{CO}_{2}$ emissions were related to air travel, a contribution similar to that of one of the campus' biggest research institutes. A return flight in economy between London and New York emits close to one metric ton of $\mathrm{CO}_{2}$ per passenger, which is around one-seventh of an average British individual's total yearly $\mathrm{CO}_{2}$ emissions and similar to the total yearly carbon footprint of the average Honduran. Multiply by the number of international conferences and attendees every year, add field trips, seminars at other institutions and visits to grant panels, and it all adds up. When the role of microorganisms in climate change is taking centre stage (Nat. Rev. Microbiol. 17, 569-586; 2019), the contributions of microbiologists should not be neglected.

There are many benefits to science gatherings beyond meeting your peers and hearing about their latest work (Front. Mar. Sci. 4, 257; 2017). These include presenting your own findings, meeting prospective students or potential mentors, establishing social networks and fostering collaborations, creating support groups, starting discussions around timely topics, and the list goes on. Conferences, particularly larger ones, also provide the opportunity for those with more limited resources and/or from more remote locations to find a larger audience with whom to discuss their findings and to learn. Conferences will not be easily replaceable by other activities and some of these benefits will be lost if they disappear, so how can one minimize their environmental impact?

There are at least two ways in which conferences can be more ecological: reduce and offset. Reducing $\mathrm{CO}_{2}$ emissions can be done in multiple ways, the easiest of which would be to fly less. As each international flight has a disproportionate impact on an individual's carbon footprint, cutting just one or two of these a year will have a major impact overall. For better established researchers that are invited to present at multiple meetings every year, skipping a couple is not likely to hinder their careers, particularly since academic productivity seems uncorrelated to the amount of air travel (Clean. Prod. 226, 959-967; 2019). Politely declining some invitations - and taking the opportunity to suggest early career researchers or minority speakers instead - would also provide opportunities for others and increase diversity in their field. Another option is to travel more ecologically, ideally by train (which produces around an order of magnitude less $\mathrm{CO}_{2}$ emissions than flights), by bus, or carpooling with others from local institutions. This will not always be possible and will undoubtedly take longer in most cases, but there are also many benefits from such 'slow travel' beyond reduced emissions (Nature 546, 565-567; 2017). Some conferences have started calculating the environmental impact for each participant and awarding 'green travel awards', a move that raises awareness.

Joining conferences remotely is another alternative. Although this impairs in-person interactions, coupled with well-structured discussion sessions and potentially subsequent online debates, these formats can provide many of the benefits of traditional meetings at a fraction of the environmental cost. There are growing numbers of 'nearly carbon-neutral' online-only conferences and instructions as to how to set them up (for example, http://bit.ly/31CsJiB). Remote joining may be particularly useful for replacing smaller gatherings, such as institutional seminars and grant or interview panels, and could enable more researchers to present their work. Another option may be to host a single event simultaneously across different locations connected remotely; this could enable people to travel locally, yet still interact in person with colleagues that live closer and 'virtually' with a larger group. This would also benefit those without the resources to travel larger distances, boost attendance by early career researchers and provide a way around travel restrictions.

A lot could be done to make current conferences more environmentally friendly, such as eliminating printed materials and other disposable items - does anyone really need another tote bag, conference pen, note pad or badge holder? Replacing poster boards with screens could eliminate singleuse posters, and providing reusable water bottles, coffee mugs and cutlery would also help - otherwise, attendees should consider bringing their own. Given the impact of meat production on greenhouse gas emissions (http://bit.ly/2KsyQyX), serving mostly or exclusively vegetarian meals can substantially cut down emissions.

Beyond reducing emissions, meetings should offer ways to offset the remaining ones. Although carbon offset schemes are increasingly common, only fewer than $5 \%$ of scientific conferences offer such options for participants (Nat. Ecol. Evol. 1, 1211-1212; 2017). As the potential cost of offsetting would be only a small fraction of the total amount spent on academic travel (for example, the offsetting cost of a LondonNew York round trip flight is around US\$30), meeting organizers should consider increasing registration fees to host carbonneutral meetings.

In a global world where science-related travel is a small fraction of a greater problem, any change to our travel habits may feel like an insignificant contribution not worth the sacrifices. However, 'flying scientists' - and 'flying editors' - emit disproportionately high amounts of pollutants compared to the average citizen, and global change is achieved by the sum of individual contributions. So, let's raise awareness to these issues and act when possible, as otherwise there may simply not be many conferences to attend in a few years.

Published online: 22 August 2019 https://doi.org/10.1038/s41564-019-0554-y 